\title{
Risk factors and clinical features of text message injuries
}

\author{
Deepak Sharan* and Ajeesh PS. \\ RECOUP Neuromusculoskeletal Rehabilitation Centre, Bangalore, India
}

\begin{abstract}
Use of mobile phone and sending text message is a very common in today's life. While sending a text message the users need to use their thumb and other palm muscles extensively. The thumb most of the time adducted on the key pad of the mobile and use high force to type the letters. Studies in literature showed that text messaging has an adverse effect on musculoskeletal system of hand. But the extensive study on the type of disorders set in among the users who extensively use mobile phone for texting. This study aims at to evaluate risk factor and clinical feature of the MSD due to hand held devices. Twenty seven subjects participated in this study. Predefined protocols were used to evaluate type of MSD occurred among the subjects. The study revealed that development of tendinitis in extensor pollicis longus, myofascial pain syndrome $(70.37 \%)$ of adductor pollicis, $1^{\text {st }}$ interossei and extensor digitorum communis. Other associated problems diagnosed were thoracic outlet syndrome (51.85\%), fibromyalgia syndrome $(25.93 \%)$, hypothyroidism $(7.41 \%)$, wrist tendinitis $(14.81 \%)$ and De Quervain's syndrome $(7.41 \%)$. It has been observed that the pathology were tendinitis of extensor pollicis longus, myofascial pain syndrome of thenar muscles and 1st interossei, extensor digitorum communis.
\end{abstract}

Keywords: "mobile phone", "texting" "musculoskeletal disorders" "risk factors"

\section{Introduction}

The use of mobile phones, other communicating devices and other hand held devices such as game controls, tablets, iPOD, PDA and keyboard have been increased in past decade [6].Text messaging, or texting, refers to the exchange of short write-up messages between fixed-line phone or mobile phone and fixed or portable devices over a network. While the original term was derived from referring to messages sent using the Short Message Service (SMS) originated from Radio Telegraphy. SMS was first used in December 1992, when Neil Papworth, a 22-year-old test engineer for Sema Group (now Airwide Solutions), used a personal computer to send the text message "Merry Christmas" via the Vodafone network to the phone of Richard Jarvis. Standard SMS messaging is limited to 140 bytes, which translates to 160 characters of the English alphabet. Initial growth of text messaging was slow, with customers in 1995 sending on average only 0.4 messages per GSM customer per month. Today, text messaging is the most widely used mobile data service, with $74 \%$ of all
* mobile phone users worldwide, or 2.4 billion out of 3.3 billion phone subscribers, at end of 2007 being active users of the Short Message Service. The advent of text messaging made possible new forms of interaction that were not possible before. A person may now carry out a conversation with another user without the constraint of being expected to reply within a short amount of time and without needing to set time aside to engage in conversation. Mobile phone users can maintain communication during situations in which a voice call is impractical, impossible, or unacceptable. Simultaneously many mobile networks have attracted people with their SMS package and different game companies introducing enthralling breath holding games which keeps consumer constantly engaging into one of these devices. Literature reported an adverse impact on the physical and psychological health of the users of mobile and other information technology [5].One year follow up study reported a high prevalence of mental symptoms in comparison to those with less use of

* Corresponding author, Email ID: deepak.sharan@recoup.in 
Information and communication technology [12]. In the same study they reported the numbers of mobile phone calls Short Message Service (SMS) text messages sent or received per day were associated with sleep disturbances, and high SMS use was also associated with symptoms of depression at follow up. They further reported use of ICT may have an impact on mental health, although the causal route yet to establish. Studies have revealed a high incidence of musculoskeletal disorders of hand, wrist, fore-arm, arm and neck has been increasing all over the world due to prolonged, forceful, low amplitude, repetitive use of such devices [3,11]. Orthopaedist and physicians have long been dealing with these patients under the name of 'Repetitive Strain Injury' or 'overuse injury'. However now these names are also been labelled as 'Nintenditis', 'Wii injury', 'SMS thumb', 'blackberry thumb', 'iPOD finger' just because of its catchiness of trendy association. Sustained and prolonged gripping, repetitive pushing, and repetitive movements with the thumb and fingers have all been identified as risk factors which may lead to disorders of the thumb and extrinsic thumb musculature in the forearm [5]. In an elaborate study, they reported median muscle activity was higher in four of the six muscles involved in texting when entering SMS messages into the mobile phone. They also found that entering an SMS message placed the thumb in abduction. The degree of abduction varies from type of mobile used by the users [5].Muscle activity while texting in mobile is also significantly different between individuals with and without musculoskeletal symptoms. A study reported that frequent triggering the physiological responses may increase muscle discomfort symptoms [8]. There are studies which revealed that design of the mobile phone and the anthropometry has an impact on the development of discomfort and fatigue in body parts like shoulder, hand etc. while using the device [2].

The aim of this study was to evaluate risk factor and clinical features of the MSD due to hand held devices.

\section{Methodology}

Twenty seven adults and one child (21 males and 7 females) between 15-50 years old were recruited from a completed retrospective experimental study [10]. Among the subjects eighteen were using blackberry, five were frequently typing in their mobile phones and one was usually playing games in the mobile phone. They were examined, diagnosed and treated in tertiary level rehabilitation clinic. The subjects with symptoms were all clinically examined according to a published protocol by a physician. After the diagnosis and assessment, all the patients underwent rehabilitation using a sequenced protocol.

\section{Results}

The type of cell phone use presented in Table 1. Table 1.

Result of type of users

\begin{tabular}{ccc}
\hline Type of mobile phone & Numbers & Percentage \\
\hline Blackberry & 21 & $77.78 \%$ \\
\hline Ordinary & 6 & $22.22 \%$ \\
\hline
\end{tabular}

The Table 1 revealed that $77.78 \%$ subjects were using Blackberry and rest of them was using ordinary cell phone. $66.67 \%$ of the blackberry users with Text Message Injury of the present study were primary level managers. $18.52 \%$ were top level CEO's of major multinational companies in India.

The results of clinical assessment of the injury or disorders and affected side of the body are presented in Table 2 and 3. Common symptoms were pain in the thumb and forearm with associated burning, numbness and tingling around the thenar aspect of the hand, stiffness of wrist and hand. Right is affected in 23 subjects; left side affected in 2 and both side affected is 2 .

Table 2 .

Results of affected body sides

\begin{tabular}{lll}
\hline Body sides affected & Numbers & Percentage \\
\hline Right side & 23 & $85.19 \%$ \\
\hline Left side & 2 & $7.41 \%$ \\
\hline Both & 2 & $7.41 \%$ \\
\hline
\end{tabular}

While doing the study it has been observed that there were a development of tendinitis in extensor pollicis longus, myofascial pain syndrome $(70.37 \%)$ of adductor pollicis, $1^{\text {st }}$ interossei and extensor digitorum communis. Other associated problems diagnosed were thoracic outlet syndrome (51.85\%), fibromyalgia syndrome $(25.93 \%)$, hypothyroidism $(7.41 \%)$, wrist tendinitis (14.81\%) and De Quervain's syndrome (7.41\%) presented in Table 3. 
Table 3.

Result of medical assessment

\begin{tabular}{lll}
\hline Type of disorders & Numbers & Percentage \\
\hline Myofascial pain syndrome & 19 & $70.37 \%$ \\
\hline Thoracic outlet syndrome & 14 & $51.85 \%$ \\
\hline Fibromyalgia syndrome & 7 & $25.93 \%$ \\
\hline Hypothyroidism & 2 & $7.41 \%$ \\
\hline Wrist tendinitis & 4 & $14.81 \%$ \\
\hline De Quervain's & 2 & $7.41 \%$ \\
\hline
\end{tabular}

\section{Discussion}

Studies related to measurement of thumb postures during SMS texting were shown to be affected by the size of the mobile phone and movement axes of the thumb [4]. Studies have revealed that while texting in mobile keypad the thumb covers motions in planes of extension, flexions, abduction-adduction and opposition. These motions occur simultaneously in three dimensions and as a result it becomes difficult to measure the kinematics of thumb [9]. Thumb movements in adduction and abduction were almost twice as fast as those in flexion or extension. It was also established that the thumb worked near the extreme range of motion, may contribute to the development of musculoskeletal disorders and this phenomena is common in the use of mobile phone while texting. On examination the pathology found were tendinitis of extensor pollicis longus, myofascial pain syndrome of thenar muscles and $1^{\text {st }}$ interossei, extensor digitorum communis and thoracic outlet syndrome in few subjects. Nintendo thumb, Gamer's grip, Nintendinitis [7] is a video game related disorder as similar to the disorders occurring in text messaging, affecting the hands. Movement of fingers are quite similar as in typing the text on mobile screen. Symptoms are like blistering, paraesthesia and swelling of the thumbs. This may affect any finger and it may lead to tendinitis, bursitis, and so on. Repeated tapping of keys while computing also leads to such ailments. In the present study similar results obtained and it is according to the available literature.

A study revealed that the postures (sitting or standing) and the type of mobile phone task (holding the phone versus texting) affected muscle activity and thumb positions [4]. In the same study they reported that females compared to males had higher muscle activity in the extensor digitorum and the abductor pollicis longus when entering SMS messages and tended to have greater thumb abduction, higher thumb movement velocities and fewer pauses in the thumb movements [4]. On the contrary in the present study such differences were not present.

The present study was only focused on twenty seven subjects. Therefore generalization of the result is difficult. As the study results showed high prevalence of myofascial pain syndrome, thoracic outlet syndrome and fibromyalgia syndrome, among the mobile phone users who are sending frequent text messaging, therefore, a study need to conduct on large sample size. The study also need to conduct on various type of mobile brand to understand the appropriateness of the design of the mobile also the user friendliness of the mobile according to the anthropometry of the users. Also it needs to further identify the pathogenesis of these disorders so that proper recommendation strategy can be formulated.

\section{References}

[1] Ahmed R.Z., UK hails 10th birthday of SMS, The Times of India., Retrieved November 20, 2008 from timesofindia.indiatimes.com/articleshow/30216466.cms

[2] Chany A.M., William S., Marras D., Burr L., The Effect of Phone Design on Upper Extremity Discomfort and Muscle Fatigue, Human Factors, 2007, 4, 602 - 618

[3] Eapen C., Bhat A.K., Prevalence Of Cumulative Trauma Disorders In Cell Phone Users, Journal of Musculoskeletal Research, 2010, 13, $137-145$

[4] Gustafsson E., Johnson P.W., Hagberg M., Thumb postures and physical loads during mobile phone use - a comparison of young adults with and without musculoskeletal symptoms, Journal of Electromyography and Kinesiology, 2010, 20, 127 -135 .

[5] Gustafsson E., Dellve L., Edlund M., Hagberg M., The use of information technology among young adults-experience, attitudes and health beliefs, Applied Ergonomics, 2003, 34, $565-570$.

[6] Jonsson P., Johnson P.W., Hagberg M. Forsman M., 'Thumb joint movement and muscular activity during mobile phone texting - A methodological study', Journal of Electromyography and Kinesiology, 2011, 21, 363-370.

[7] Koh T.H., Ulcerative "nintendinitis": a new kind of repetitive strain injury, Med J Aust, 2000, 173, 671. 
[8] Lin I-Mei and Peper E., Psychophysiological Patterns During Cell Phone Text Messaging: A Preliminary Study, Applied Psychophysiologycal Biofeedback, 2009, 34, 53-57.

[9] Ong F.R., Thumb Motion and Typing Forces during Text Messaging on a Mobile Phone, 13th International Conference on Biomedical Engineering, IFMBE Proceedings, 2009, 23, $2095-2098$.

[10] Sasidharan A.P., Sharan D., Rameshkumar R., Risk Factors, Clinical Features and Outcome of Treatment of Work Related Musculoskeletal Disorders in On-Site Clinics in Indian IT Companies, Grahamstown, South Africa, ODAM 2011.

[11] Sharan D., 2011, personal communication, 13 May.

[12] Thomée S., Eklöf M., Gustafsson E., Nilsson R., Hagberg M., Prevalence of perceived stress, symptoms of depression and sleep disturbances in relation to information and communication technology (ICT) use among young adults - an explorative prospective study, Computers in Human Behavior, $2007,23,1300-1321$. 